\title{
Electron diffraction studies of supersonic jets. VII. Liquid and plastic crystalline carbon tetrachloride
}

\author{
Edward J. Valente and Lawrence S. Bartell \\ Department of Chemistry, University of Michigan, Ann Arbor, Michigan 48109
}

(Received 28 September 1983; accepted 4 October 1983)

\begin{abstract}
Carbon tetrachloride at 3-11 mol \% in neon carrier produces clusters of $\mathrm{CCl}_{4}$ molecules in flow through a Laval nozzle. Electron diffraction patterns of the clusters formed at a total pressure below 2.5 bar are intermediate between $\mathrm{x}$-ray diffraction patterns of bulk liquid $\mathrm{CCl}_{4}$ and neutron patterns of the amorphous material formed by condensation at $10 \mathrm{~K}$. Strong, sharp interference features at low angle imply appreciable long range order, perhaps beyond $50 \AA$, in the clusters, whose temperatures are about $210 \mathrm{~K}$. When carrier partial pressure is increased beyond $2.6 \mathrm{bar}$, clusters begin to exhibit crystallinity. Crystallites belong to the rhombohedral plastic-crystalline phase $\mathrm{Ib}, a=14.27 \AA$ and $\alpha=90.0^{\circ}$.
\end{abstract}

\section{INTRODUCTION}

Condensed carbon tetrachloride can exist in several phases, including liquid, amorphous, plastic crystalline, and several ordered crystalline phases. Each of these has been the subject of recent diffraction investigations. A neutron diffraction study of amorphous $\mathrm{CCl}_{4}$ formed by condensation onto a cold $(10 \mathrm{~K})$ surface indicates a structure with order extending beyond $20 \AA$, and strong maxima in the pair correlation function at 3.8 and $6.0 \AA$ associated with chlorine contacts between adjacent and interlocked molecules. ${ }^{1}$ At $293 \mathrm{~K}$, neutron and $\mathrm{x}$-ray diffraction patterns of bulk liquid $\mathrm{CCl}_{4}$ display similar well defined maxima and analysis shows that strong correlations exist between molecules in the first coordination sphere. ${ }^{2,3}$ Longer range correlations probably present were not addressed or fully accessible to the studies by conventional diffraction techniques. Dispersive $\mathrm{X}$-ray diffraction by liquid $\mathrm{CCl}_{4}$ between 253 and $336 \mathrm{~K}$ confirms that longer range ordering is enhanced by lower temperatures, as manifested by a narrowing of and an increase in the intensity of the lowest angle diffraction feature $\left(s=1.3 \AA^{-1}\right){ }^{4}$ Diffraction patterns show fine structure at lower angle, and the general pattern is ascribed to a local body-centered cubic cluster $\operatorname{model}^{5}(a=5.8 \AA)$. At $250 \mathrm{~K}$, $\mathrm{CCl}_{4}$ crystallizes as a plastic crystalline face-centered cubic structure Ia and below $226 \mathrm{~K}$ undergoes a transition to an ordered monoclinic form. ${ }^{6}$ A plastic crystalline rhombohedral phase Ib is also known and is stable in the same temperature range as the fcc form. ${ }^{6,7}$ Other solid phases have also been identified at high external pressures. ${ }^{8}$ Transitions between liquid and the fcc and rhombohedral plastic crystal phases have been observed by diffractometry ${ }^{9}$ and calorimetry. ${ }^{10}$

In earlier papers in this series, we described the formation of liquid-like clusters of benzene in supersonic expansions seeded at low mole fractions into monatomic carrier gases. ${ }^{11,12}$ Electron diffraction patterns were shown to have good sensitivity and a high angular resolving power able to characterize sharp low-angle features displayed by systems with long-range order. Such resolving power is of particular relevance in a study of $\mathrm{CCl}_{4}$. In a prior investigation ${ }^{13}$ of liquid $\mathrm{CCl}_{4}$ it had been pointed out that the resolving power of conventional $\mathrm{x}$-ray and neutron methods is inadequate to demonstrate the full extent of ordering in the condensed material. On the other hand, the long-range order reported in this novel energy dispersive $\mathrm{x}$-ray study of liquid $\mathrm{CCl}_{4}$ seemed remarkable. Because this result depended crucially on the sharpness of one small-angle feature that could not be displayed in the same runs that monitored the rest of the pattern, it seemed worthwhile to apply a more conventional diffraction approach but one offering the resolution afforded by electron-optical techniques. The existence of several computer simulations of condensed $\mathrm{CCl}_{4}$ added additional incentive to carry out an electron diffraction study of the substance. In the sections following, we describe the results obtained when electrons are scattered by condensed $\mathrm{CCl}_{4}$ formed in nozzle flow.

\section{EXPERIMENTAL}

We have described previously the apparatus and methods employed in producing clusters by expansions of seeded jets of monatomic carriers in Laval nozzles. ${ }^{14}$ All work presented in the following was done with glass nozzle No. $6 .{ }^{15}$ Carbon tetrachloride was A.C.S. reagent grade, and neon (99.99\%) was obtained from Air Products Ltd.; both were

TABLE I. Experimental conditions.

\begin{tabular}{cllll}
\hline \hline & & \multicolumn{3}{c}{ Rel. } \\
Run $^{\mathrm{a}}$ & $\boldsymbol{X}_{\mathrm{CCl}}$ & $\boldsymbol{P}_{\text {tot }}{ }^{\mathrm{b}}$ & \multicolumn{1}{c}{ exposure $^{\mathrm{c}, \mathrm{d}}$} & \multicolumn{1}{c}{ Plate no. $^{\mathrm{d}}$} \\
\hline$\ldots$ & 1.0 & 0.12 & $3.2(95)$ & $787-790(940-943)$ \\
$\ldots$ & 1.0 & 0.12 & $(60)$ & $(777-780,980-983)$ \\
2a & 0.05 & 1.4 & $0.30(17)$ & $928-930(924,925)$ \\
2b & 0.09 & 1.4 & $0.30(5.7)$ & $792,793(784,785)$ \\
2c & 0.09 & 1.4 & $0.36(7.1)$ & $906,908(912,913,917,918)$ \\
3a & 0.06 & 2.2 & 0.15 & 804 \\
3b & 0.05 & 2.7 & 0.13 & 805 \\
3c & 0.038 & 3.4 & 0.11 & 794 \\
3d & 0.043 & 3.7 & 0.07 & 204 \\
3e & 0.033 & 4.8 & 0.07 & 206 \\
\hline \hline
\end{tabular}

"Designations refer to labels in Figs. 2 and 3. Carrier, neon.

b Total stagnation pressure, in bar.

c Time in $s$ to produce pattern of unit absorbance with $100 \mathrm{nA}$ electron beam.

${ }^{d}$ Parentheses, $r^{2}$ sector; without parentheses, $r^{1}$ sector. 
used without further purification. Neon was passed over liquid $\mathrm{CCl}_{4}$ in a brass tank at room temperature $(301 \mathrm{~K})$ containing a glass wool plug in contact with the $\mathrm{CCl}_{4}$ to serve as a wick. Electron diffraction patterns of the effluent vapor were recorded on Kodak $4 \times 5$ in. medium projector slides with $40 \mathrm{keV}$ electrons $(\lambda=.061 \AA)$. Plates exposed under the same experimental conditions were averaged, and all intensities were leveled with aid of scattering factors taken from Sellers et al. ${ }^{16}$ Intensities for representative plates are available as supplementary material. ${ }^{17}$ Expansion and exposure conditions are given in Table I.

\section{DIFFRACTION INTENSITIES}

The studies presented here represent a preliminary examination of $\mathrm{CCl}_{4}$ cluster formation in neon carrier. Neat $\mathrm{CCl}_{4}$ does not condense on expansion through Laval nozzle No. 6. Molecular diffraction patterns of neat $\mathrm{CCl}_{4}$ disclose monomer scattering only [Fig. 1(a)]. A systematic residual [Fig. 1(b)] observed after subtraction of the theoretical molecular intensity from the experimental intensity is probably due principally to the standard theoretical simplification embodied in the independent atom model. ${ }^{18}$ When $\mathrm{CCl}_{4}$ at 0.14 bar is expanded with neon at a total stagnation pressure of $1.4 \mathrm{bar}$, clusters of $\mathrm{CCl}_{4}$ form and the resultant cluster beam is far more highly collimated than neat $\mathrm{CCl}_{4}$ jets as evidenced by the deposition of a small $(9 \mathrm{~mm})$ button of condensed $\mathrm{CCl}_{4}$ on the cold trap $120 \mathrm{~mm}$ downstream of the skimmer. Conspicuous modification of the diffraction intensity from that of the monomer scattering is evident in Fig. 1(c). Cluster signals were obtained from the total intensity by subtraction of the monomer intensity, a scaled, leveled intensity of the carrier gas, and a smooth background polynomial.

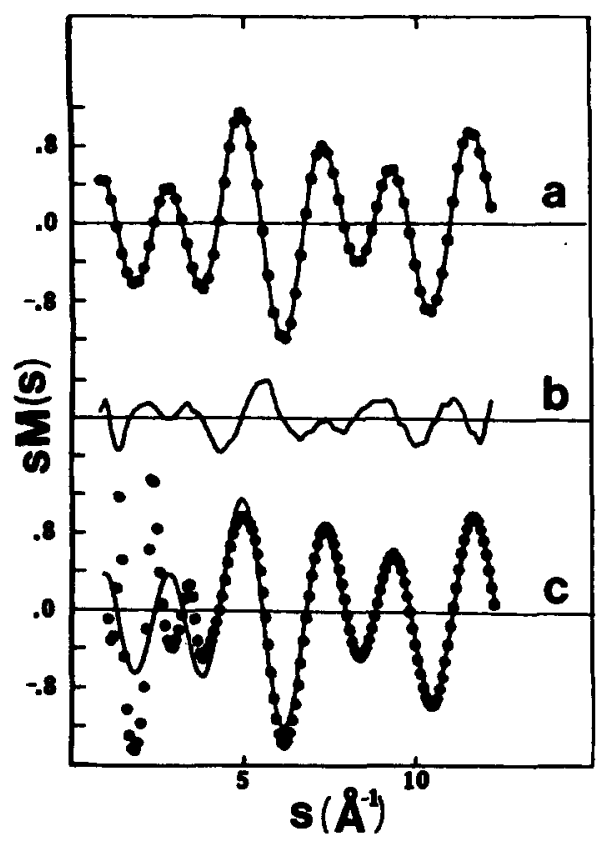

FIG. 1. Reduced intensity curves: (a) Pure $\mathrm{CCl}_{4}$. Points, experimental $(0.12$ bar). Solid line, theoretical monomer. (b) Systematic difference between the experimental and theoretical curves multiplied by 8 (see the text). (c) Points, experimental data for $\mathrm{CCl}_{4}(0.12 \mathrm{bar})$ with neon carrier (1.3 bar). Solid trace, theoretical curve for $\mathrm{CCl}_{4}$ monomer. Note the strong cluster signal at small scattering angle.

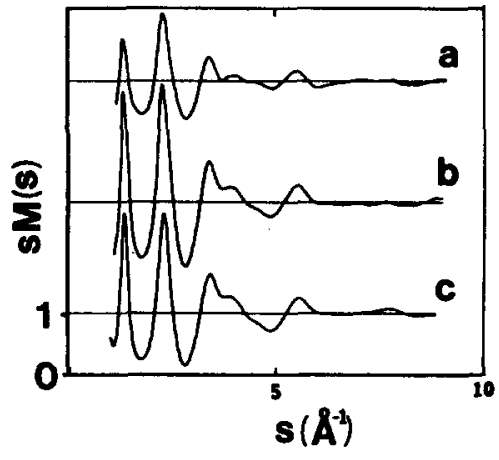

FIG. 2. Intermolecular scattering by $\mathrm{CCl}_{4}$ clusters; conditions of Table I.

Sampling was at $\Delta s \leqslant \pi / 40$, consistent with the sharpness of the features in the cluster pattern.

Monomer intensity was based on the structure as described previously ${ }^{19,20}$ with amplitudes of vibration from Morino et al. ${ }^{20} \mathrm{~A}$ scale factor was refined in least squares to absorb the uncertainty in our camera height. Although the cluster beam is well collimated, it is still broader than the gas jets from tubular nozzles in standard electron diffraction studies, and the additional damping produced was accounted for as described previously. ${ }^{12}$ Data measured through our $r^{2}$ and $r^{1}$ rotating sectors were merged, and the small systematic residual consistently observed in the neat $\mathrm{CCl}_{4}$ experiments was subtracted. Typical cluster intensities for the $r^{1}+r^{2}$ combined range are shown in Fig. 2 .

As the presure of the carrier is increased while maintaining constant partial pressure of $\mathrm{CCl}_{4}$, the smooth features characteristic of the cluster signal just described begin to display irregularities that grow into sharp crystal lines. This transition begins at 2.7 bar total pressure and $\mathrm{CCl}_{4}$ mole fraction 0.05 . A sequence illustrating the changes in the molecular intensity over the low angle $\left(r^{1}\right)$ range as the carrier pressure is increased is in Fig. 3.

\section{DISCUSSION}

Most prominent in the diffracted intensity of $\mathrm{CCl}_{4}$ clusters at lower carrier pressure are the low angle maxima at

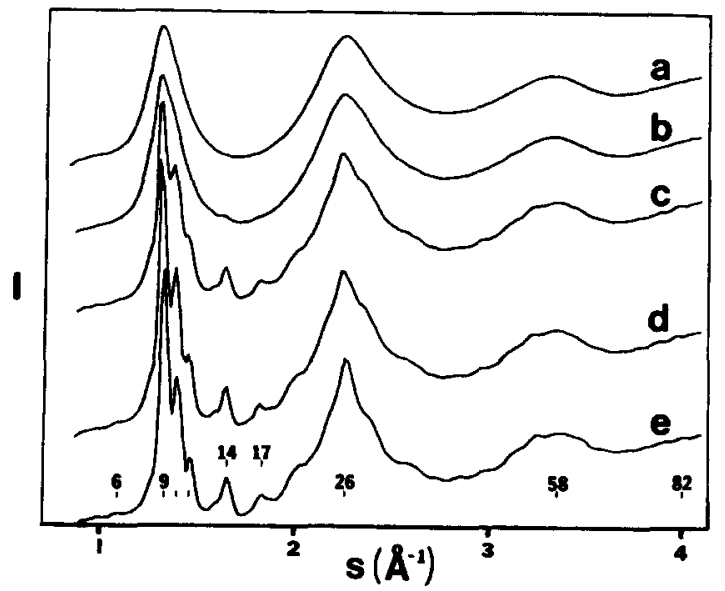

FIG. 3. Leveled intensities of electrons scattered by clusters generated from saturated $\mathrm{CCl}_{4}$ vapor in neon carrier at total pressures, bar, of (a) 2.2, (b) 2.7, (c) 3.4, (d) 3.7 and (e) 4.8. Inset values of $h^{2}+k^{2}+l^{2}$ for reflections from a pseudocubic cell. 
$s=1.3$ and $2.3 \AA^{-1}$. Compared with the corresponding $\mathrm{x}$ ray diffraction features for the bulk liquid, ${ }^{2}$ these are better resolved and displaced slightly outward. General aspects of our patterns bear a closer resemblance to those of the neutron diffraction study of amorphous $\mathrm{CCl}_{4}$ at $10 \mathrm{~K}$. ${ }^{1}$

Long range order in liquid $\mathrm{CCl}_{4}$ is indicated by the maximum near $s=1.3 \AA^{-1}$. Neutron diffraction by $\mathrm{CCl}_{4}$ at room temperature and at $10 \mathrm{~K}^{1}$ has lower resolution than the room temperature angle dispersion $\mathrm{x}$-ray studies ${ }^{2}$ and, hence, the low angle maxima are broader. Energy-dispersive $x$-ray diffraction experiments ${ }^{4}$ gave $\mathrm{CCl}_{4}$ patterns having sharper low-angle maxima than obtained by angle dispersion. Electron diffraction ring breadths more nearly resemble those of the energy-dispersive $x$-ray method than those found in the standard diffraction experiments. We observe the FWHM of the peak at $s=1.3 \AA^{-1}$ to be $0.18 \AA^{-1}$, as compared to $0.30 \AA^{-1}$ for the energy dispersive $\mathrm{x}$-ray work. Colder temperatures in the cluster beam as well as higher resolving power no doubt account for this difference. Resolution afforded by the conventional electron diffraction method is not only adequate to characterize the small-angle features just discussed but also can readily measure much finer features when they arise (see Fig. 3). Inasmuch as an analysis of the energy dispersive $\mathrm{x}$-ray pattern indicated substantial ordering beyond $30 \AA$ in liquid $\mathrm{CCl}_{4}$, an ordering beyond $50 \AA$ is implied for our clusters by the ring width of our patterns (3/5 that of Ref. 5).

A rough estimation of the temperature of the $\mathrm{CCl}_{4}$ clusters may be made from the position of the low angle maximum. At room temperature ( $298 \mathrm{~K}$ ), the average of observations is $s=1.29 \AA^{-1}$, and at $10 \mathrm{~K}$ the amorphous structure shows the feature at $1.40 \AA^{-1}$. We observe it at $1.35 \AA^{-1}$, a position indicating a structure with density and temperature between these extremes. A change in density of $\mathrm{CCl}_{4}$ liquid as the cube of the peak position predicts a $28 \%$ increase in density from 298 to $10 \mathrm{~K}$ in reasonable agreement with experiment.' Similarly for cluster $\mathrm{CCl}_{4}$ an $11 \%$ density increase is implied. By extrapolating normal liquid density ${ }^{21}$ of $\mathrm{CCl}_{4}$ in the range 343 to $293 \mathrm{~K}$ down to the apparent amorphous value, we obtain a temperature of about $210 \mathrm{~K}$ for the $\mathrm{CCl}_{4}$ clusters.

Features of the liquid-like pattern of plate No. 804 [Fig. 3(a)] taken with $\mathrm{CCl}_{4}$ mole fraction 0.06 at 2.2 bar total pressure are almost identical to those described above for mole fraction 0.10 and total pressure $1.4 \mathrm{bar}$ [Fig. 2(c)]. When the stagnation pressure is increased to $2.7 \mathrm{bar}$, however, traces of Debye-Scherrer rings begin to appear in the feature at $s=1.3 \AA^{-1}$. Above $3.4 \mathrm{bar}$, the first amorphous feature is well resolved into powder diffraction rings. Crystallite sizes inferred from the breadths of the crystal lines appear to be about $100 \AA$ in diameter. Diffuse halos at higher angle are marked by the ripples of unresolved powder rings, suggesting a mixture or that the long range order is of the plastic crystalline type, and that rotational disorder is still present in the $\mathrm{CCl}_{4}$ clusters.

Powder ring radii correspond to the more stable rhombohedral plastic-crystalline phase of $\mathrm{CCl}_{4}, \mathrm{Ib}$, and the lines can be indexed from $n^{2}=4-82$ (hkl: 200-910, 833) for the pseudocubic cell with $a=14.27 \AA, \alpha=90^{\circ}$. The cell repeat distance is shorter than that found for rhombohedral $\mathrm{CCl}_{4}$ at $233 \mathrm{~K}$, and the density of $1.85 \mathrm{~g} / \mathrm{cm}^{3}$ is close to that found for the ordered monoclinic phase near $225 \mathrm{~K} .^{6}$

Previously, plastic-crystalline phases Ia and Ib have been observed by $x$-ray diffractometry at $243 \mathrm{~K}$ to give prominent powder rings and diffuse liquid features. ${ }^{22}$ These features were associated with the large domains of the polycrystals and therefore crystal orientation effects. Rotation of the sample gave a diffuse pattern clearly differing from that of the liquid, however. Our phase $\mathrm{Ib} \mathrm{CCl}_{4}$ clusters are, of course, randomly oriented. We do not know the extent to which the $\mathrm{CCl}_{4}$ clusters as sampled by the electron beam may have been modified after leaving the nozzle, since the structure of the shock wave in the vicinity of the skimmer has not been established. If any cluster phase Ia had been formed it might not have survived annealing during passage through a Mach disk. ${ }^{23} \mathrm{~A}$ transition from liquid-like to crystalline condensate as a function of the mole fraction of $\mathrm{CCl}_{4}$ and the carrier pressure can be understood, in part, in terms of the theory of homogeneous nucleation in nozzle flow. Increasing the carrier pressure at constant partial pressure of $\mathrm{CCl}_{4}$ in simulations ${ }^{11}$ leads to lower temperatures at the region of onset of nucleation. Whether the additional turbulence contributes to equilibrium, i.e., to the avoidance of the supercooling found at lower pressure, is not known.

We have not sought to analyze the structure of condensed $\mathrm{CCl}_{4}$ from electron diffraction patterns of its clusters. The strong similarity between the present patterns and those obtained from $\mathrm{x}$-ray and neutron diffraction indicates that amorphous $\mathrm{CCl}_{4}$ clusters are structurally similar to the bulk liquid and amorphous solid at $10 \mathrm{~K}$. Structures of these phases have already been discussed extensively. ${ }^{1-5}$ It is worthwhile mentioning that the structure of the cold, dense, amorphous solid was reasonably well represented by the reference interaction site model (RISM), a model constructed to treat the structure of liquids. One systematic imperfection in the RISM representation of $\mathrm{CCl}_{4}$ seems to be, according to the newer high resolution diffraction patterns, that RISM underestimates the full extent of the long-range ordering. A second discrepancy noted for other tetrahedral molecules ${ }^{24}$ and for cold benzene ${ }^{11}$ is that RISM can greatly underestimate the orientational correlations between adjacent molecules.

Our study shows that nozzle flow techniques can produce condensed phases of various types with particle sizes large enough to correspond to bulk material yet small enough to be amenable to electron diffraction. Furthermore, liquid-like phases can be generated at temperatures not easily achieved in other types of experiments with far different time scales, and novel solid phases ${ }^{15}$ can be induced to form as well. These facts, together with the high beam intensities and resolution offered by electrons make electron diffraction studies of clusters a promising tool for the investigation of condensed matter.

\section{ACKNOWLEDGMENTS}

This research was supported by a grant from the National Science Foundation. We thank Mr. John Shanahan 
for assistance with experiments. We gratefully acknowledge a generous allocation of computing time from the Michigan Computing Center.

'M. R. Chowdhury and J. C. Dore, J. Non-Cryst. Solids 46, 343 (1981).

${ }^{2}$ A. H. Narten, J. Chem. Phys. 65, 573 (1976).

${ }^{3}$ G. Reichelt, J. U. Weidner, and N. Zimmermann, Ber. Bunsenges. Phys. Chem. 78, 1050 (1974).

${ }^{4}$ K. Nishikawa, K. Tohji, M. Shima, and Y. Murata, Chem. Phys. Lett. 64, 154 (1979).

${ }^{5}$ K. Nishikawa and Y. Murata, Bull. Chem. Soc. Jpn, 52, 293 (1979).

${ }^{6} R$. Rudman and B. Post, Science 154, 1009 (1966).

'J. Badiali, J. Bruneaux-Poulle, and A. Defrain, J. Chim. Phys. 73, 113 (1976).

${ }^{8}$ G. J. Piermarini and A. B. Braun, J. Chem. Phys. 58, 1974 (1973).

${ }^{9}$ K. Tohji, K. Nishikawa, and Y. Murata, Jpn. J. Appl. Phys. 19, L365 (1980).

10 J. A. Morrison and E. L. Richards, J. Chem. Thermodyn. 8, 1033 (1976).

"E. J. Valente and L. S. Bartell, J. Chem. Phys. 80 (1984).

${ }^{12}$ R. K. Heenan, E. J. Valente, and L. S. Bartell, J. Chem. Phys. 78, 243 (1983).

${ }^{13}$ Y. Murata and K. Nishikawa, Bull. Chem. Soc. Jpn. 51, 411 (1978).

${ }^{14}$ L. S. Bartell, R. K. Heenan, and M. Nagashima, J. Chem. Phys, 78, 236 (1983).

${ }^{15}$ E. J. Valente and L. S. Bartell, J. Chem. Phys. 79, 2683 (1983).
${ }^{16}$ H. L. Sellers, L. Schafer, and R. A. Ronham, J. Mol. Struct. 49, 125 (1978).

${ }^{17}$ See AIP document no. PAPS JCPSA-80-1458-18 for 18 pages of tables containing experimental intensities, background functions, and intermolecular scattering for plates discussed in this work. Order by PAPS number and journal reference from American Institute of Physics, Physics Auxiliary Publication Servics, 335 East 45th Street, New York, N. Y. 10017. The price is $\$ 1.50$ for each microfiche ( 98 pages) or $\$ 5.00$ for photocopies of up to 30 pages, and $\$ 0.15$ for each additional page over 30 pages. Air mail additional. Makes checks payable to the American Institute of Physics.

${ }^{18}$ P. Pulay, R. Mawhorter, D. A. Kohl, and M. Fink, J. Chem. Phys. 79, 185 (1983); S. R. Goates and L. S. Bartell, ibid. 77, 1866, 1874 (1982).

${ }^{19}$ L. S. Bartell, L. O. Brockway, and R. H. Schwendeman, J. Chem. Phys. 23, 1854 (1953); J. Haase and W. Zeil, Z. Phys. Chem. Neue Folge 45, 202 (1965); I. Hargittai, J. Herciadi, and J. Tremmel, Jeuaer Ruudschau 13, 3 (1968); S. Shibata, K. Iijima, R. Taui, and Y. Nakamura, Rep. Fac. Sci. Shizuoka Univ. 9, 33 (1974).

${ }^{20}$ Y. Morino, Y. Nakamura, and T. Iijima, J. Chem. Phys. 32, 643 (1960).

${ }^{21}$ V. M. Kozhin, Sov. Phys. Crystallogr. 14, 630 (1969).

${ }^{22}$ K. Nishikawa and K. Tohji, J. Chem. Phys. 74, 5817 (1981).

${ }^{23}$ G. Torchet, J. Farges, M. F. de Feraudy, and B. Raoult, Rarefied Gas Dynamics, edited by R. Campargue, (Commissariat a l'Energie Atomique, Paris, 1979), Vol. 11, p. 1175.

${ }^{24}$ D. G. Montague, M. R. Chowdhury, J. C. Dore, and J. Reed, Mol. Phys. 50, 1 (1983). 